\title{
Extramural Venous Invasion in Patients with Locally Advanced Esophageal Cancer: A Reminder to Pathologists to Look Harder
}

\author{
Catherine J. Streutker, MD, MSc ${ }^{1,2}$ \\ ${ }^{1}$ Department of Laboratory Medicine, St. Michael's Hospital, Toronto, Canada; ${ }^{2}$ Department of Laboratory Medicine and \\ Pathobiology, University of Toronto, Toronto, ON, Canada
}

Venous invasion (VI), the infiltration of malignancies into veins, has been described as an important predictor of patient outcome in a variety of tumors, i.e., renal, ${ }^{1}$ colorectal, ${ }^{2}$ pancreas, ${ }^{3}$ and stomach ${ }^{4}$. In contrast to lymphatic space invasion, which tends to predict lymph node involvement, VI appears to predict distant metastases, particularly to the liver. ${ }^{5}$ The issue has perhaps been best studied in renal carcinoma, where hilar vein involvement is routinely reported, and in colorectal carcinomas, where papers on the subject date back to the 1930 s. ${ }^{6}$ Extramural VI (EMVI), involving veins in the pericolonic fat in pathological stage T3 colorectal carcinomas, should be identified in at least $30 \%$ of cases; ${ }^{7}$ however, for many years, colorectal carcinoma EMVI was not well reported by pathologists, even those subspecializing in gastrointestinal tract pathology. Although the older College of American Pathologists (CAP) protocol for evaluation of colorectal cancers included a recommendation to look for both EMVI and intramural VI (IMVI), in the synoptic protocols developed in the early 2000s there was only one line item for 'lymphovascular space invasion', combining lymphatic space invasion, EMVI and IMVI into one factor, with no allowance for details. For most pathologists, observation of VI when it was clearly obvious on hematoxylin and eosin (H\&E)-stained slides would merit a comment on this, however few pathologists would have made any particular extra effort to identify EMVI. Thus, as Quirke and Morris noted in 2007, the reported rate of VI for colorectal carcinoma languished at approximately $10 \%$, rather than

\footnotetext{
(C) Society of Surgical Oncology 2018
}

First Received: 25 March 2018;

Published Online: 10 April 2018

C. J. Streutker, MD, MSc

e-mail: Streutkerc@smh.ca
$30 \% .^{7}$ Comments such as this led to a renewed interest in better detection of VI. EMVI is now listed as a line item in the most current CAP cancer reporting protocols (see ww w.cap.org/web/home/protocolsandguidelines/cancerreport ingtools) and is a specific item in the UK's Royal College of Pathologists cancer datasets (see www.rcpath.org/profe ssion/publications/cancer-datasets.html).

Several studies have evaluated the utility of elastic stains (i.e., Movat's, Elastica van Gieson) to better identify sites of VI. ${ }^{8,9}$ Having participated in one of these studies, ${ }^{2}$ it was readily evident that the detection of VI on H\&Estained slides was not as simple as we had previously understood it to be. We thought that the clues of 'orphan arterioles' and 'protruding tongues' were sufficient to detect EMVI. Unfortunately, it is extremely easy to miss VI in veins that have been so destroyed by the invasive tumor that there is no clearly evident venous wall remaining on the H\&E-stained slide. It was humbling to see how many more foci of VI could be found on an elastic stain in cases where there had been no suspicion at all for VI on the H\&E-stained slides. Whether one does elastic stains on all tumor blocks or chooses to do the stains on blocks with suspicious findings on H\&E-stained slides, it has become clear that pathologists should be optimizing VI detection in this way for colorectal carcinomas.

This interest in the detection of VI has now, appropriately, spread to other gastrointestinal tract tumors. In this issue of Annals of Surgical Oncology, the publication by Faiz et al. ${ }^{10}$ details the utilization of both H\&E and elastic stains to evaluate the rate of EMVI in esophageal carcinomas. As with colorectal carcinomas, EMVI was identified in a significant proportion of malignancies, but was considerably higher in squamous carcinomas (42.9\%) than adenocarcinomas $(16.7 \%)$ in the surgery-alone group. In this surgery-only group, the presence of EMVI was a significant predictor of disease-free survival (DFS) and 
overall survival (median DFS for EMVI-/node- was 79 months, and 32 months for EMVI+/node-). Interestingly, although the rate of finding EMVI in patients who had received neoadjuvant therapy prior to surgery was similar to that in the surgery-alone group, EMVI did not predict survival in the post-neoadjuvant therapy group. This was a relatively small and therefore underpowered group that may have been affected by factors that affect choice of neoadjuvant therapy versus surgery alone, particularly the fact that some of these post-therapy tumors may have originally been higher-stage tumors. The authors note that larger studies of EMVI in post-neoadjuvant therapy tumors are needed. A limitation of this study is the methodology of choosing slides for elastin staining. Rather than using suspicion of VI on H\&E-stained slides to guide further testing, a better method is to either stain multiple blocks with elastin stains as an initial stain without performing an H\&E-stained slide at all, or performing minimal pre-screening with $\mathrm{H} \& \mathrm{E}$-stained slides to select for testing those with invasion past the muscularis propria. Castonguay et al. ${ }^{11}$ demonstrated that, in esophageal adenocarcinomas, VI was frequently detected in cases that had previously been signed out as negative when using only H\&E-stained slides. Despite these factors, this publication raises the visibility of an often underrecognized prognostic feature in gastrointestinal malignancies, particularly for a location (esophagus) where the literature on EMVI is still limited. Considering that a recent meta-analysis also demonstrated prognostic significance for colonic IMVI ${ }^{12}$, further studies on the possible prognostic utility of IMVI in the esophagus would also be of importance. Finding appropriate prognostic factors in this area is needed since the ever-increasing use of mucosal resection techniques in early esophageal adenocarcinomas leads to persistent problems in the selection of patients with early submucosal invasion for either surveillance or surgery.

\section{REFERENCES}

1. Nwadei IU, Lorentz A, Patil D, et al. Renal cell carcinoma with vena caval involvement; a contemporary clinicopathologic analysis of 53 case. Hum Pathol. 2016;49:83-9.

2. Kirsch R, Messenger DE, Riddell RH, et al. Venous invasion in colorectal cancer; impact of an elastin stain on detection and interobserver agreement among gastrointestinal and nongastrointestinal pathologists. AJSP. 2013;37;200-10.

3. Mierke F, Hempel S, Distler $M$ et al. Impact of portal vein involvement from pancreatic cancer on metastatic pattern after surgical resection. Ann Surg Oncol. 2016;23 Suppl 5;730-36.

4. Lee EW, Lee WY, Koo HS. Prognostic factors for node-negative advanced gastric cancer after curative gastrectomy. J Gastric Cancer. 2016;16(3):161-6.

5. Talbot IC, Ritchie S, Leighton MH, et al. The clinical significance of invasion of veins by rectal cancer. $\mathrm{Br} J$ Surg. 1980;67(6):439.

6. Brown CF, Warren S. Visceral metastasis from rectal carcinoma. Surg Gynecol Obstet. 1938;66:11-21.

7. Quirke P, Morris E. Reporting colorectal cancer. Histopathology. 2007;50:103-12.

8. Howlett CJ, Tweedie EJ, Driman DK. Use of an elastic stain to show venous invasion in colorectal carcinoma: a simple technique for detection of an important prognostic factor. $J$ Clin Pathol. 2009;62:1021-5.

9. Sato T, Ueno H, Mochizuki $\mathrm{H}$, et al. Objective criteria for the grading of venous invasion in colorectal cancer. AJSP. 2010;34:454-62.

10. Faiz Z, Huygen LJW, Alquethami HJ, et al. Prevalence and prognostic significance of extramural venous invasion in patients with locally advanced esophageal cancer. Ann Surg Oncol. 2018. https://doi.org/10.1245/s10434-018-6448-z.

11. Castonguay MC, Li-Chang HH, Driman DK. Venous invasion in oesophageal adenocarcinoma; enhanced detection using elastic stain and association with adverse histologic features and clinical outcomes. Histopathology. 2014;64(5):693-700.

12. Knijn N, van Exsel UEM, de Noo ME, Nagetgaall ID. The value of intramural vascular invasion in colorectal cancer: a systematic review and meta-analysis. Histopathology. 2018;72(5):721-8. 